\title{
A comparison of antibody titres in mouse uterine fluid after immunization by several routes, and the effect of the uterus on antibody titres in vaginal fluid
}

\author{
E. L. Parr and M. B. Parr \\ Department of Anatomy, School of Medicine, Southern Illinois University, Carbondale, \\ Illinois 62901-6503, USA
}

\begin{abstract}
Summary. Measurements of specific antibody titres in uterine fluid of mice immunized by different routes indicated that two immunizations in the pelvic presacral space using aluminium hydroxide as adjuvant was a simple and effective way to elicit a significant IgA and IgG response. Higher IgA and IgG titres were produced in uterine fluid by subcutaneous immunization with antigen in Freund's complete adjuvant followed by intravaginal boosting without adjuvant, but this immunization involved both a toxic adjuvant and repeated applications of large doses of antigen in the vagina. Intragastric immunization produced an IgA response in the uterus but no IgG. Local intravaginal priming and boosting with large doses of antigen without adjuvant produced an $\operatorname{IgA}$ response in uterine fluid, but was less effective for IgG and was inefficient in terms of time and the amount of antigen used.

Hysterectomy reduced the concentration of specific IgA in vaginal fluid of immunized mice to no more than $5 \%$ of normal, indicating that most of the IgA in vaginal fluid originates in the uterus. In contrast, IgG titres were not significantly different in hysterectomized and intact mice. IgA titres in vaginal fluid were at least partly restored to normal levels in sham-hysterectomized mice.
\end{abstract}

Keywords: mucosal immunity; uterus; vagina; vaccination; mouse

\section{Introduction}

Secretions of the female reproductive tract contain antibodies of both the IgA and IgG classes. These antibodies may be important in defence against infections of the reproductive tract, and they could potentially be useful for immunocontraception. The amount of antibody in uterine fluid is regulated by steroid hormones, with larger amounts of both immunoglobulin classes being present in rat uterine fluid during oestrus or oestradiol treatment than during dioestrus or progesterone treatment (Wira \& Sullivan, 1982). In the secretions of other mucosal tissues, including the mouse vagina (Parr et al., 1988), the titre of specific antibody is influenced by the route of immunization, with local application of antigen to the mucosal surface often being most effective in stimulating specific IgA secretion (Waldman \& Ganguly, 1974; Bienenstock, 1986). However, data on specific antibody titres in uterine fluid after immunization by different routes are not available for any species. In the present study we developed two methods for collection of uterine fluid samples from mice, and measured specific anti-ferritin IgA and IgG titres in these fluids after immunization with horse ferritin by several routes.

We also investigated the contribution of uterine fluid antibody to the antibody which is present in vaginal fluid. Antibody in vaginal fluid may originate in serum or vaginal plasma cells and cross the vaginal mucosa directly, or it may be secreted into uterine fluid and later drain into the vagina. Early quantitative measurements indicated that IgA and IgG concentrations in the vaginal fluid of 
3 hysterectomized women were essentially the same as in normal women (Waldman et al, 1971). However, a larger study by Jalanti \& Isliker (1977) indicated that the concentration of IgA in vaginal fluid of hysterectomized women was only about $10 \%$ of normal, while IgG was present at normal levels. This suggested that IgG might cross the vaginal mucosa, while IgA mainly entered the vagina from the uterus. Raffi et al. (1977) reported that IgA and IgG were both present in vaginal fluid of hysterectomized women, and they suggested that both immunoglobulins were probably synthesized locally in vaginal mucosa and/or transported across this mucosa from serum. Specific IgA and IgG antibodies against $E$. coli were demonstrated in vaginal fluid of hysterectomized women by Stamey et al. (1978). The studies of Raffi et al. (1977) and Stamey et al. (1978) were qualitative, and did not indicate whether vaginal fluid immunoglobulin concentrations in the hysterectomized women were at normal levels. Wira \& Sullivan (1985) reported that the content of IgA in rat vaginal fluid was low when its content in uterine fluid was high, and they suggested that different regulatory mechanisms operate at the two sites. The vagina is lined by stratified squamous epithelium, and secretion of IgA across such epithelium has not been reported. However, secretory component, the receptor for IgA transport, has been demonstrated in rat vaginal epithelium at prooestrus (Parr \& Parr, 1989), raising the possibility that IgA may be secreted locally across the vaginal mucosa in some species. To determine the contribution of the uterus to vaginal fluid antibody, we measured specific anti-ferritin IgA and IgG titres in vaginal fluid of immunized mice that were either hysterectomized (no contribution from the uterus) or intact (normal contribution from the uterus).

\section{Materials and Methods}

Female ICR mice 60-100 days old were purchased from Sasco, Inc. (Omaha, NE, USA). The animals were kept in a light cycle of $12 \mathrm{~h}$ light: $12 \mathrm{~h}$ dark, and were housed adjacent to males of the same strain to induce cycling during intravaginal immunization and vaginal fluid collection.

Mice were immunized with horse ferritin using 4 procedures singly or in combination: (i) subcutaneously (s.c.) near the scapulae with $50 \mu \mathrm{g}$ horse ferritin (Calbiochem, La Jolla, CA, USA; cadmium-free) in complete Freund's adjuvant (Difco, Inc., Detroit, MI, USA), (ii) intravaginally (i.vag.) by daily insertion for 10 consecutive days of cotton tampons containing $50 \mu \mathrm{l}$ ferritin at $10 \mathrm{mg} / \mathrm{ml}$ without adjuvant, (iii) by intragastric (i.g.) administration of $5 \mathrm{mg}$ ferritin in $0.5 \mathrm{ml}$ of $0.2 \mathrm{M}$-sodium bicarbonate by feeding needle, or (iv) intramuscularly with $50 \mu \mathrm{g}$ ferritin adsorbed to $50 \mu \mathrm{g}$ aluminium hydroxide (Alu-gel-S ${ }^{\circledR}$, Serva Feinbiochemica, Heidelberg, FRG) in the muscle that is attached to the ventral surface of the sacrum (presacral space, p.s.). For this injection the needle started between the base of the tail and the anus and passed about $8 \mathrm{~mm}$ toward the sacrum. Six groups of 5-6 mice were immunized as follows: s.c./i.vag., i.g., i.g./i.vag., i.vag., i.vag./i.vag., and p.s./p.s. A common schedule of immunization and uterine fiuid collection was used for all mice: primary immunization on Day 1 (s.c., i.g., p.s., or the first of 10 days of i.vag.), ovariectomy on Day 17, i.vag. boosting if applicable on Days 21-31 or p.s. boosting on Day 25, stimulation with $1 \mu \mathrm{g}$ depot oestradiol cypionate in peanut oil (s.c.; The Upjohn Co., Kalamazoo, MI, USA) and cervical ligation on Day 32 , and uterine fluid collection on Day 35. About 50-100 $\mu$ l clear fluid was present in the uterine lumen, and care was taken to avoid blood contamination when it was collected. Uterine fluid was collected from non-immunized mice for use as a control, and from additional mice immunized s.c./i.vag. for use as a standard. Another group of mice (p.s./p.s./x) was immunized in the presacral space as above, but instead of ovariectomy and oestradiol stimulation to induce the accumulation of uterine fluid, the uterine body was removed from each mouse on Day 25 leaving the uterine horns in place. The cut ends of the uterine horns healed closed, and 50-100 $\mu \mathrm{l}$ clear fluid were collected from them on Day 35. This group of mice was housed adjacent to males to induce cycling after the partial hysterectomy.

Two experiments were done to study the influence of hysterectomy on antibody in vaginal fluid. In the first, 3 groups of 10 mice each were hysterectomized, sham hysterectomized, or left intact on Day 1 . All mice were immunized s.c. as described above on Day 9 and were boosted i.vag. as described above. Vaginal fluid was collected daily on Days $42-45$ by pipetting $50 \mu \mathrm{l}$ phosphate-buffered saline (PBS) in and out of the vagina several times. Particulate matter was removed from the washings by centrifugation, and the supernatants were stored at $-20^{\circ} \mathrm{C}$. The 4 vaginal washings from each mouse were pooled for antibody measurements. Vaginal fluid was collected from 10 intact, non-immunized mice to serve as a control. In the second experiment, 3 groups of 10 mice each were immunized p.s. as described above on Day 1. On Day 24 the mice were hysterectomized, sham hysterectomized, or left intact, and on Day 36 they were boosted p.s. with the same dose of vaccine. Vaginal fluid was collected daily on Days 50-53 as described above, and also from 10 hysterectomized, non-immunized mice to serve as a control.

Hysterectomies were done under anaesthesia induced by tribromoethanol. The cervix was transected near the pubic symphysis and the distal cut end was ligated. The proximal part of the cervix, the uterine body, and both uterine horns were removed, leaving the ovaries in place. At the time of necropsy the vagina and adjacent cervix were 
carefully removed and fixed by immersion in neutral buffered $10 \%$ formalin. When the tissue became firm, serial longitudinal sections were cut with a razor blade and examined with a dissecting microscope. It was found that the cut end of the cervix had healed closed in all cases, and that the site of transection was always in the unbranched portion of the cervical canal, which is lined by stratified squamous epithelium (Parr \& Parr, 1985). No part of the uterine body remained attached to the vagina. Sham hysterectomies were done as a control to confirm that reconnection of the uterus to the vagina would restore vaginal fluid antibody titres to near normal levels. The cervix was transected as for hysterectomy, but the cut ends were then rejoined and held in place by sutures through the myometrium. At the time of necropsy, trypan blue dye was injected into a uterine horn and its appearance in the vagina confirmed that cervical anastomosis was effective.

Antibody titres in uterine and vaginal fluid were measured by ELISA. The following reagents were added to the wells of microtitre plates (Immulon 1, Dynatech Laboratories, Inc., Alexandria, VA, USA) at room temperature with intermediate washings in PBS containing 0.05\% Tween 20 (PBS-Tween): $50 \mu \mathrm{l}$ ferritin or bovine serum albumin (BSA) at $25 \mu \mathrm{g} / \mathrm{ml}$ in 0.10 M-carbonate buffer ( $\mathrm{pH} \mathrm{9.5)} \mathrm{overnight;} 2 \%$ calf serum in PBS-Tween for $30 \mathrm{~min}$, followed by $25 \mu \mathrm{l}$ of serial $2 \cdot 25$-fold dilutions of uterine fluid or vaginal washings overnight; $50 \mu \mathrm{l}$ of a 1:2000 dilution of horseradish peroxidase (HRP)-conjugated rabbit anti-mouse IgA or IgG ( $\alpha$ - or $\gamma$-chain specific, Zymed, San Francisco, CA, USA) for $2 \mathrm{~h}$; and $100 \mu \mathrm{l}$ tetramethylbenzidine (Miles Scientific, Naperville, IL, USA) at $0.5 \mathrm{mg} / \mathrm{ml}$ in $0.10 \mathrm{M}$-acetate citrate buffer $(\mathrm{pH} \mathrm{6.0)}$ containing $0.005 \%$ hydrogen peroxide for $1 \mathrm{~h}$. The enzyme reactions were stopped with an equal volume of $1.0 \mathrm{M}$-sulphuric acid and the absorbances of the wells were measured at $450 \mathrm{~nm}$ in a Titer Tek ELISA plate reader.

Sample titres are defined as the reciprocal of the highest dilution with a net absorbance (ferritin minus BSA) of greater than $0 \cdot 3$. The most concentrated samples were $1 / 2.25^{2}$ dilutions (two $2 \cdot 25$-fold dilutions) of neat uterine fluid or $1 / 2.25$ dilutions of vaginal washings. If the net absorbance at these dilutions was less than 0.3 the sample was assigned a titre of $2 \cdot 25^{1}$ (uterine fluid) or $2 \cdot 25^{\circ}$ (vaginal washings). These titres represent antibody concentrations that were below the limit of detectability. Geometric mean titres defined as: $\log _{2 \cdot 25}$ geometric mean titre $=1 / n\left(\log _{2 \cdot 25}\right.$ titre $1+\log _{2.25}$ titre $2+\ldots+\log _{225}$ titre $n$ ) were calculated, and the statistical significance of differences was determined by $t$ tests. Vaginal fluid antibody titres in Exps 1 and 2 were all measured at the same time and should therefore be closely comparable. Uterine fluid antibody titres are comparable because a standard immune uterine fluid was included in each measurement. Before sample titres were determined, their absorbances were corrected by the proportion that the absorbance of the standard on that day differed from its mean; corrected sample titres rarely differed from uncorrected titres.

The percentage of vaginal fluid immunoglobulin secreted locally across the vaginal mucosa is given by: \% local secretion $=C_{h} / C_{i} \times 100 \%$, where $C_{h}$ and $C_{i}$ are the mean immunoglobulin concentrations in vaginal fluid of hysterectomized and intact mice, respectively. These concentrations can be written $\mathrm{C}_{\mathrm{h}}=\mathrm{C}_{\mathrm{o}} \times \mathrm{gmh}$ and $\mathrm{C}_{\mathrm{i}}=\mathrm{C}_{\mathrm{o}} \times$ gmi, where $C_{o}$ is the immunoglobulin concentration when the geometric mean antibody titre is $2.25^{\circ}$ and gmh and gmi are the measured geometric mean titres of the hysterectomized and intact mice. Thus, percentage local secretion is given by $\mathrm{gmh} / \mathrm{gmi} \times 100 \%$. Most sample titres in the hysterectomy group were $2 \cdot 25^{\circ}$. Because such antibody concentrations are below the limit of detectability and may actually be less than $1 / 2.25 \times$ their concentration when the titre is $2 \cdot 25^{\prime}$, the calculation gives an upper limit for percentage local secretion.

\section{Results}

\section{Antibody titres in uterine fluid}

Specific antibody titres in mouse uterine fluid after immunization by several routes are shown in Fig. 1. The highest IgG titre was produced by subcutaneous priming with antigen in complete Freund's adjuvant followed by i.vag. boosting without adjuvant (s.c./i.vag.). A relatively high IgG titre was also observed in animals primed and boosted in the presacral space with antigen adsorbed to aluminium hydroxide (p.s./p.s.). Intravaginal application of repeated large doses of antigen without adjuvant caused relatively smaller IgG titres (i.vag. and i.vag./i.vag.), while intragastric administration of a single large dose of antigen without adjuvant produced no IgG response (i.g.). The highest IgA titre was produced by the s.c./i.vag. and i.vag./i.vag. routes, and several other routes of immunization produced titres that were nearly as high, including i.g./i.vag., i.g., and p.s./p.s. Control mice had no specific antibody in uterine fluid (ni). Antibody titres were similar in uterine fluids collected by two different methods from mice that were immunized the same way (p.s./p.s. and p.s./p.s./x).

\section{Effect of uterus on antibody in vaginal fluid}

Hysterectomy caused mean vaginal fluid IgA titres to decline nearly to $2 \cdot 25^{\circ}$ with both s.c./ i.vag. and p.s./p.s. routes of immunization (Table 1). The upper limit of vaginal fluid IgA secreted 


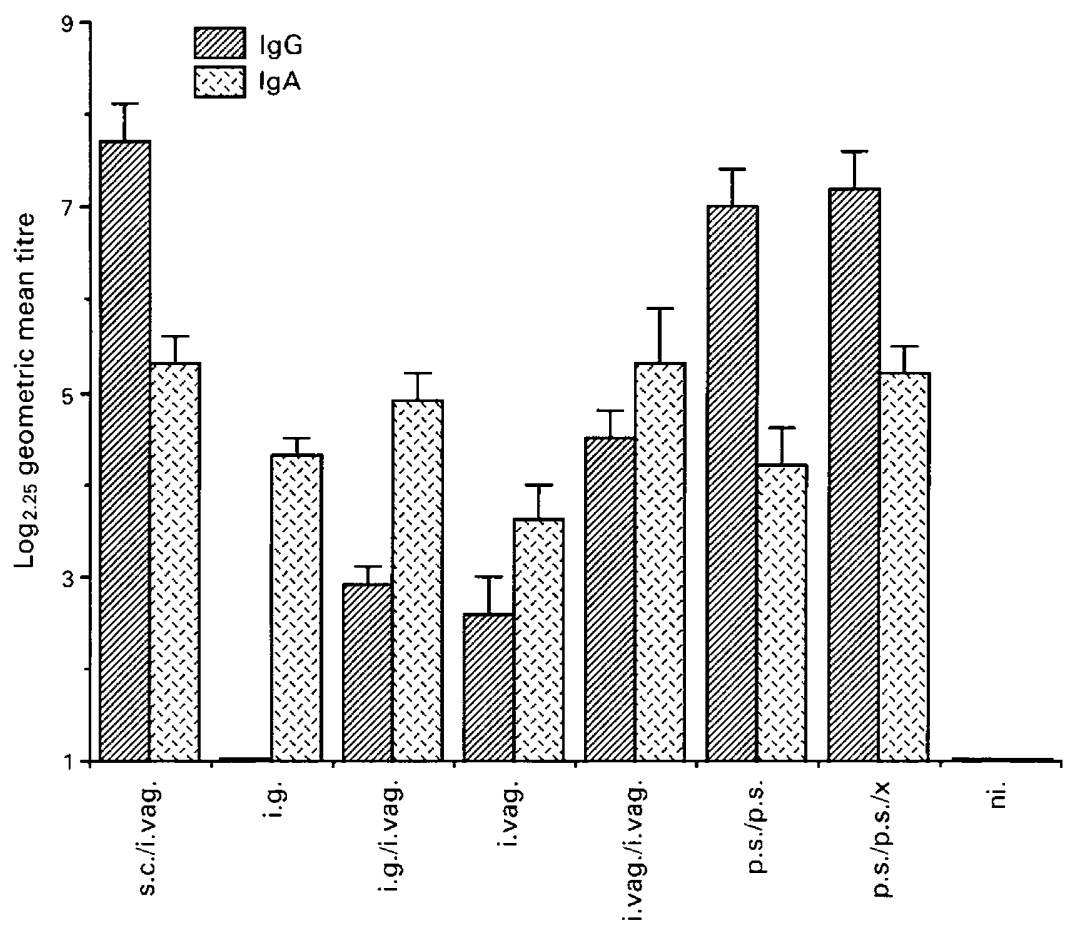

Fig. 1. $\log _{2.25}$ geometric mean antibody titres in mouse uterine fluid after immunization with horse ferritin by several routes. The routes of immunization were: subcutaneous priming near the scapulae followed by intravaginal boosting (s.c./i.vag.), intragastric (i.g.), intragastric priming followed by intravaginal boosting (i.g./i.vag.), intravaginal (i.vag.), intravaginal priming and boosting (i.vag./i.vag.), priming and boosting in the pelvic presacral space (p.s./p.s.), priming and boosting in the presacral space followed by uterine fluid collection by the partial hysterectomy method (p.s./p.s./x), and non-immune mice (ni.). There were 5 mice in the i.g./i.vag. and i.vag./i.vag. groups, and 6 mice in all other groups. Vertical bars indicate standard errors of the mean.

locally across the vaginal mucosa was $6.3 \%$ in Exp. 1 (s.c./i.vag.) and 3.9\% in Exp. 2 (p.s./p.s.). The titre data thus indicate that at least $95 \%$ of the IgA in vaginal fluid of cycling mice is derived from the uterus. The ELISA absorbance data indicate that a small amount of specific IgA is present in vaginal fluid. The mean ELISA absorbance of the most concentrated samples in the hysterectomy group of Exp. 1 was $0.17 \pm 0.03$, while in the non-immune control group it was $0.04 \pm 0.02$. In Exp. 2, the mean ELISA absorbance of the most concentrated samples in the hysterectomy group was $0.26 \pm 0.03$, while in the non-immune control group it was $0.02 \pm 0.02$. The differences are significant $(P<0.005$ and $P<0.0005$ respectively) and indicate that a small amount of $\operatorname{IgA}$ is secreted locally across the vaginal mucosa. Hysterectomy caused mean vaginal fluid IgG titres to increase by one dilution in both experiments (Table 1), but the differences were not significant at the $5 \%$ level in either case. In the sham-hysterectomy groups there was a partial restoration of both IgA and IgG titres in vaginal fluid to levels seen in intact mice.

\section{Discussion}

An optimal immunization protocol to elicit humoral immune responses in luminal fluids of the female reproductive tract has not yet been determined, and may vary with the individual antigen, adjuvant, isotype of antibody desired, and the purpose of the immunization. Routes of 
Table 1. Effect of hysterectomy on antibody titres in vaginal fluid of immunized mice

\begin{tabular}{lcccc}
\hline $\begin{array}{l}\text { Route of } \\
\text { immunization }\end{array}$ & Isotype & Intact & Hysterectomized & $\begin{array}{c}\text { Sham } \\
\text { hysterectomized }\end{array}$ \\
\hline s.c./i.vag. & A & $3 \cdot 8 \pm 0 \cdot 7$ & $0 \cdot 4 \pm 0 \cdot 3$ & $3 \cdot 0 \pm 0 \cdot 7$ \\
& G & $9 \cdot 2 \pm 0 \cdot 5$ & $10 \cdot 2 \pm 0 \cdot 3$ & $9 \cdot 3 \pm 0 \cdot 5$ \\
p.s./p.s. & A & $4 \cdot 3 \pm 0 \cdot 6$ & $0 \cdot 3 \pm 0 \cdot 1$ & $1 \cdot 4 \pm 0.4$ \\
& G & $6 \cdot 4 \pm 0 \cdot 5$ & $7 \cdot 4 \pm 0 \cdot 5$ & $5 \cdot 9 \pm 0.7$ \\
\hline
\end{tabular}

Antibody titres are expressed as $\log _{2.25}$ geometric mean titre \pm s.e.m.

immunization used in the present study were chosen largely on the basis of responses observed previously in vaginal fluid (Parr et al., 1988; Thapar et al., 1990a, 1990b), and on a desire to maximize IgA responses because this immunoglobulin class is more protective than IgG in the intestine and respiratory tract (Fubara \& Freter, 1973; Bessen \& Fischetti, 1988). Two immunizations in the pelvic presacral space with ferritin adsorbed to aluminium hydroxide was an effective way to stimulate an IgA and IgG response in mouse uterine fluid. The occurrence of a significant IgA response in uterine fluid after non-mucosal immunization in the presacral space is consistent with other results in our laboratory showing that immunization at this site stimulated significantly higher IgA titres in vaginal fluid than did subcutaneous immunization near the scapulae with the same vaccine (Thapar et al., 1990b). The immunological basis of IgA secretion in uterine fluid after immunization in the presacral space is not known, but should be investigated. In comparison, subcutaneous priming and intravaginal boosting produced higher antibody titres, but involved both a more potent adjuvant and repeated applications of large doses of antigen in the vagina during boosting. Intravaginal priming and boosting elicited a comparable $\operatorname{IgA}$ response but again involved repeated applications of large doses of antigen. Immune responses resulting from fewer applications or smaller doses of intravaginal ferritin have not been studied in detail, but preliminary observations suggest that the responses are smaller. Moreover, the effectiveness of intravaginal immunization was probably enhanced in these mice by their ovariectomy, which would have led to a thinner vaginal epithelium at the time of boosting. In other studies we have found that movement of proteins across the vaginal mucosa after intraluminal administration was detectable at metoestrus, dioestrus and pro-oestrus, when the epithelium is thinnest, but not at oestrus (Parr \& Parr, 1990). Stimulation of the intestinal immune system with a single large intragastric dose of ferritin without adjuvant produced an IgA response in uterine fluid not no IgG. Non-replicating antigens such as ferritin seldom stimulate vigorous immune responses when administered in the intestine (Waldman \& Ganguly, 1974; Fuhrman \& Cebra, 1981; Nicklin \& Miller, 1983; Elson \& Ealding, 1984; Dahlgren et al., 1986). Our results therefore suggest that progress in increasing the effectiveness of intestinal vaccines could have implications for immunity in the reproductive tract.

The uterine fluid used for the present measurements was only an approximation of normal uterine fluid since its collection involved hormonal and/or surgical intervention. However, the fact that similar IgA and IgG titres were observed in uterine fluids collected by two different methods from mice immunized the same way (p.s./p.s. and p.s./p.s./x) suggests that the measurements may be representative of immune responses in true uterine fluid. The partial hysterectomy method was easier to perform than the ovariectomy method, provided a larger volume of uterine fluid with increasing time, and its fluid contained slightly higher antibody titres.

Hysterectomy caused a marked reduction of specific IgA titres in vaginal fluid of immunized mice, indicating that at least $95 \%$ of the IgA in mouse vaginal fluid originates in the uterus. Since the hysterectomies were done 42 days or 50 days before vaginal fluid was sampled, it seems unlikely that our results are an artefact of surgical trauma. Anastomosis of the cut ends of the cervix in the sham-hysterectomized animals partly restored vaginal fluid IgA titres to normal. Failure to achieve complete restoration of IgA titres may have been due to effects of chronic inflammation around the 
cervical sutures on this immunoglobulin as it passed through the affected region. The small amount of specific IgA that was present in vaginal fluid of hysterectomized mice apparently crossed the mucosa of the vagina or the adjacent cervix. It might have crossed the mucosa as a passive transudate from serum or local plasma cells, or it might have been actively transported by secretory component. The cervix and vagina are both lined by stratified squamous epithelium in mice (Parr \& Parr, 1985), and active transport of $\operatorname{IgA}$ across such an epithelium has not previously been reported. However, we detected secretory component in rat vaginal epithelium at pro-oestrus (Parr \& Parr, 1989), and a small amount of active transport remains a possibility. The IgA probably did not enter the vagina from the peritoneal cavity because the cervical transection was healed closed in all cases, and it was not due to a small remnant of uterine body attached to the cervicovaginal portion of the tract. Our data are consistent with measurements showing that IgA concentrations in vaginal fluid of hysterectomized women were only about $10 \%$ of normal (Jalanti \& Isliker, 1977). Hysterectomy increased the IgG titre in vaginal fluid by $2 \cdot 25$-fold with both routes of immunization, but the increases were not statistically significant in either case. The data indicate that essentially all of the IgG in vaginal fluid crosses the vaginal mucosa.

The observation that most of the IgA in mouse vaginal fluid is passively acquired from the uterus implies that measurements of IgA in vaginal fluid, if carried out over a sufficient time in cycling animals (see below), can give an indication of IgA secretion in the uterus. Moreover, passively administered specific $\operatorname{IgA}$ has been reported to be more protective against mucosal infection than an equivalent or larger amount of specific IgG in both the intestine (Vibrio cholera: Fubara \& Freter, 1973) and respiratory tract (Streptococcus pyogenes: Bessen \& Fischetti, 1988). IgA may therefore play a role in resistance to infections of the cervix, vagina and vulva, even though it is not significantly secreted across those mucosae.

The regulation of immunoglobulins in uterine and vaginal secretions by sex steroid hormones has been extensively studied. Wira \& Sandoe (1980) and Wira \& Sullivan (1985) reported that rat uterine IgA and IgG content were increased by 3 days of oestradiol treatment, whereas vaginal IgA and IgG were reduced by the same treatment. The authors suggested that the mechanisms controlling the amounts of immunoglobulins in the uterus and vagina are different. Armstrong (1968) demonstrated that oestradiol treatment caused fluid retention in the rat uterine lumen by closure of the cervix. Our results indicate that the uterus is the source of nearly all IgA in the mouse vagina, and if this applies in the rat, it can be suggested that oestradiol increases uterine and decreases vaginal IgA mainly by causing cervical closure. Vaginal fluid IgG, on the other hand, is a mixture of uterine and vaginal contributions. Oestradiol treatment would block the uterine component by closure of the cervix, and might reduce transudation across the vaginal mucosa by causing the vaginal epithelium to become thicker.

We thank Shonah Hunter for technical assistance and Virginia Rendleman and Candida Morris for typing the manuscript. This work was supported by NIH grant HD-17337.

\section{References}

Armstrong, D.T. (1968) Hormonal control of uterine luminal fluid retention in the rat. Am.J. Physiol. 214, $764-771$.

Bessen, D. \& Fischetti, V.A. (1988) Passive acquired mucosal immunity to group A streptococci by secretory immunoglobulin A. J. exp. Med. 167, 19451950.

Bienenstock, J. (1986) IgA synthesis and stimulation. In Progress Towards Better Vaccines, pp. 39-47. Eds R. Bell \& G. Torrigiani. Oxford University Press, Oxford.

Dahlgren, U. I.H., Ahlstedt, S. \& Hanson, L.A. (1986) Origin and kinetics of IgA, IgG and IgM milk anti- bodies in primary and secondary responses of rats. Scand. J. Immunol. 23, 273-278.

Elson, C.O. \& Ealding, W. (1984) Generalized systemic and mucosal immunity in mice after mucosal stimulation with cholera toxin. J. Immunol. 132, 27362741 .

Fubara, E.S. \& Freter, R. (1973) Protection against enteric bacterial infection by secretory IgA antibodies. J. Immunol. 111, 395-403.

Fuhrman, J.A. \& Cebra, J.J. (1981) Special features of the priming process for a secretory IgA response: $B$ cell priming with cholera toxin. J. exp. Med. 153, 534-544.

Downloaded from Bioscientifica.com at 04/26/2023 10:06:03AM 
Jalanti, R. \& Isliker, H. (1977) Immunoglobulins in human cervico-vaginal secretions. Int. Archs Allergy appl. Immunol. 53, 402-408.

Nicklin, S. \& Miller, K. (1983) Local and systemic immune responses to intestinally presented antigen. Int . Archs Allergy appl. Immunol. 42, 87-90.

Parr, E.L., Parr, M.B. \& Thapar, M. (1988) A comparison of specific antibody responses in mouse vaginal fluid after immunization by several routes. J. Reprod. Immunol. 14, 165-176.

Parr, M.B. \& Parr, E.L. (1985) Immunohistochemical localization of immunoglobulins $A, G$, and $M$ in the mouse female genital tract. J. Reprod. Fert. 74, 361-370.

Parr, M.B. \& Parr, E.L. (1989) Immunohistochemical investigation of secretory component and immunoglobulin $\mathrm{A}$ in the genital tract of the female rat. $J$. Reprod. Fert. 85, 105-113.

Parr, M.B. \& Parr, E.L. (1990) Antigen recognition in the female reproductive tract: I. Uptake of intraluminal protein tracers in the mouse vagina. J. Reprod. Immunol. In press.

Raffi, R.O., Moghissi, K.S. \& Sacco, A.G. (1977) Proteins of human vaginal fluid. Fert. Steril. 28, 13451348.

Stamey, T.A., Wehner, N., Mihara, G. \& Condy, M. (1978) The immunologic basis of recurrent bacteriuria: role of cervico-vaginal antibody in enterobacterial colonization of the introital mucosa. Medicine $57,47-56$.
Thapar, M., Parr, E.L. \& Parr, M.B. (1990a) Immune responses in mouse vaginal fluid after intravaginal immunization: the effect of adjuvants. J. Reprod. Immunol. (in press).

Thapar, M., Parr, E.L. \& Parr, M.B. (1990b) Secretory immune responses in mouse vaginal fluid after pelvic, parenteral, or vaginal immunization. Immunology (in press).

Waldman, R.H. \& Ganguly, R. (1974) Techniques for eliciting mucosal immune response. Acta endocr., Copenh., Suppl. 194, 262-277.

Waldman, R.H., Cruz, J.M. \& Rowe, D.S. (1971) Immunoglobulin levels and antibody to Candida albicans in human cervicovaginal secretions. Clin. exp. Immunol. 9, 427-434.

Wira, C.R. \& Sandoe, C.P. (1980) Hormonal regulation of immunoglobulins: influence of oestradiol on immunoglobulins $\mathrm{A}$ and $\mathrm{G}$ in the rat uterus. Endocrinology 106, 1020-1026.

Wira, C.R. \& Sullivan, D.A. (1982) Effect of oestradiol and progesterone on the secretory immune system in the female genital tract. Adv. exp. Med. Biol. 138, 99-106.

Wira, C.R. \& Sullivan, D.A. (1985) Oestradiol and progesterone regulation of IgA, IgG and secretory component in cervico-vaginal secretions of rat. Biol. Reprod. 32, 90-97.

Received 7 November 1989 